\title{
Partial molar pregnancy and coexisting fetus with Turner syndrome: Case report and literature review
}

\author{
Ji Eun Park', Ji Kwon Park', ${ }^{1, *}$ In Ae Cho², and Jong Chul Baek ${ }^{1}$ \\ 'Department of Obstetrics and Gynecology, Gyeongsang National University Changwon Hospital, Gyeongsang National University College of \\ Medicine, Changwon, Korea \\ ${ }^{2}$ Department of Obstetrics and Gynecology, Gyeongsang National University Hospital, Gyeongsang National University College of Medicine, \\ Jinju, Korea
}

\begin{abstract}
Partial hydatidiform mole and coexisting fetus is a rare entity with antecedent high risk of maternal and fetal complications, and risk of persistent trophoblastic disease in later life. Here, we report a case of twin pregnancy with live fetus identified as 45, X and normal placenta and another partial mole. Ultrasound scan at 10 weeks showed a hydrops fetus with a focal area of multicystic placenta. The patient underwent chorionic villus sampling and amniocentesis for chromosomal analysis, and the result was 45,X. Based on these finding, the patient then underwent induced abortion. Pathological examination (immunohistochemical staining) of the placenta confirmed the partial mole. This report suggests that careful prenatal ultrasonography and appropriate karyotyping in a molar pregnancy and coexisting fetus enable early diagnosis and may be beneficial for prognosis.
\end{abstract}

Key words: Hydatidiform mole, Twins, Ultrasonography, Karyotyping.

\section{Introduction}

A hydatidiform mole coexistent with a live fetus is rare, with an incidence of one in 22,000 pregnancies [1]. A molar pregnancy results from a genetic error during the fertilization process, and three different mechanisms may underlie the formation of hydatidiform mole with a coexistent live fetus: complete mole (diploid, all paternal in origin) coexistent with a normal diploid fetus, partial mole (triploid, maternal, and/or paternal in origin) coexistent with a normal diploid fetus, and partial mole with an abnormal triploid fetus (both having 69 chromosomes of maternal and paternal origin) [2]. It is even rarer for the fetus to coexist with a partial versus complete mole [3].
Here, we report a case of twin pregnancy with partial mole and coexisting hydropic fetus, identified as $45, X$, which occurred following ovulation induction that ended at 11 weeks of gestation. The diagnostic and management approaches for such cases are discussed, along with a review of literature.

\section{Case}

A 37-year-old nulliparous woman was referred for consultation at 10 and 3/7 weeks of gestation due to fetal cystic hygroma. She had conceived with twin pregnancy following intrauterine insemination, but one fetus miscarried at 9 and $3 / 7$ weeks of gestation (26 mm embryo, no heart motion) and

Received: February 21 2018, Revised: March 25 2018, Accepted: March 26 2018, Published: 30 June 2018

*Corresponding author: Ji Kwon Park, M.D., Ph.D. (iD http://orcid.org/0000-0002-9731-190X

Department of Obstetrics and Gynecology, Gyeongsang National University Changwon Hospital, Gyeongsang National University College of Medicine, 11 Samjeongja-ro, Seongsan-gu, Changwon 51472, Korea.

Tel: +82-55-214-3780, Fax: +82-55-214-3253, E-mail: obgypjk@gnu.ac.kr

Conflict of interest: The authors declare that they do not have any conflicts of interest.

(c) This is an open-access article distributed under the terms of the Creative Commons Attribution Non-Commercial License (http://creativecommons.org/licenses/by-nc/4.0/) which permits unrestricted non-commercial use, distribution, and reproduction in any medium, provided the original work is properly cited.

(c) Copyright 2018 by the Korean Society of Medical Genetics and Genomics 
the above findings of the other fetus were observed. There was no personal or familial history of genetic abnormalities. She reported taking no teratogenic medications, and there was no recent viral infection and no diabetes mellitus or hypertension. Her husband was 38 years old. She and her husband were nonconsanguineous and appeared to be healthy. They did not have a family history of congenital malformation.

A fetal ultrasound at Gyeongsang National University Hospital indicated live fetal biometry consistent with gestational age (11 and 2/7 weeks). The nuchal translucency was $8.93 \mathrm{~mm}$ and bilateral pleural effusion was seen. Moreover, an area in the placenta corresponding to the demised fetus containing multiple small cystic structures without blood flow mixed with normal placenta was identified (Fig. 1).

The above findings suggested the possibility of chromosomal abnormalities of the fetus and molar pregnancy. The prognosis of fetal hydrops and risk of pregnancy maintenance accompanied by hydatidiform mole potential were thoroughly discussed with the mother, and it was decided to terminate the pregnancy. We performed both chorionic villus sampling and early amniocentesis for karyotyping. Two methods were used for karyotyping to prevent misdiagnosis due to confined placental mosaicism. The titer of serum human chorionic gonadotropin (hCG) was above 50,000 IU/L. Conventional karyotyping and fluorescence in situ hybridization (FISH) analyses were performed using cells obtained from chorionic villi and amniotic fluid. All of the above tests indicated a fetal karyotype of 45,X (Fig. 2). Induced abortion was performed at 11 and 5/7 weeks of gestation.
Pathological study showed a dichorionic, diamniotic intervening membrane at the border between the two placentas. Separate placental tissues measuring $8 \times 5 \times 2 \mathrm{~cm}$ and $7.5 \times 4.5 \times 3 \mathrm{~cm}$ were detected. In the latter placenta, a separate container containing grossly visible vesicles was observed. Histological examination confirmed partial hydatidiform mole with hydropic chorionic villi and trophoblast inclusions. Immunohistochemical staining indicated the expression of p53, distinguishing partial hydatidiform mole from hydropic abortion, and of p57, confirming partial hydatidiform mole rather than complete mole (Fig. 3). The patient was followed up with serial serum beta hCG monitoring, which returned to the normal level within 3 months. Chest Xray and thyroid function test results were normal. She showed no signs of persistent trophoblastic disease at 2-year follow-up.

\section{Discussion}

Hydatidiform moles are abnormal pregnancies characterized by varying degrees of trophoblastic proliferation, and vesicular swelling of placental villi associated with an absent or abnormal fetus/embryo that can be transferred to a gestational trophoblastic tumor [4]. Hydatidiform moles are categorized into complete and partial types based on gross morphology, histopathological examination, and karyotype [5].

Complete moles are almost always diploid following monospermic fertilization of an anuclear empty ovum with genomic endoreduplication or dispermic fertilization of an anuclear empty ovum. In both cases, the chromosome in the complete mole is
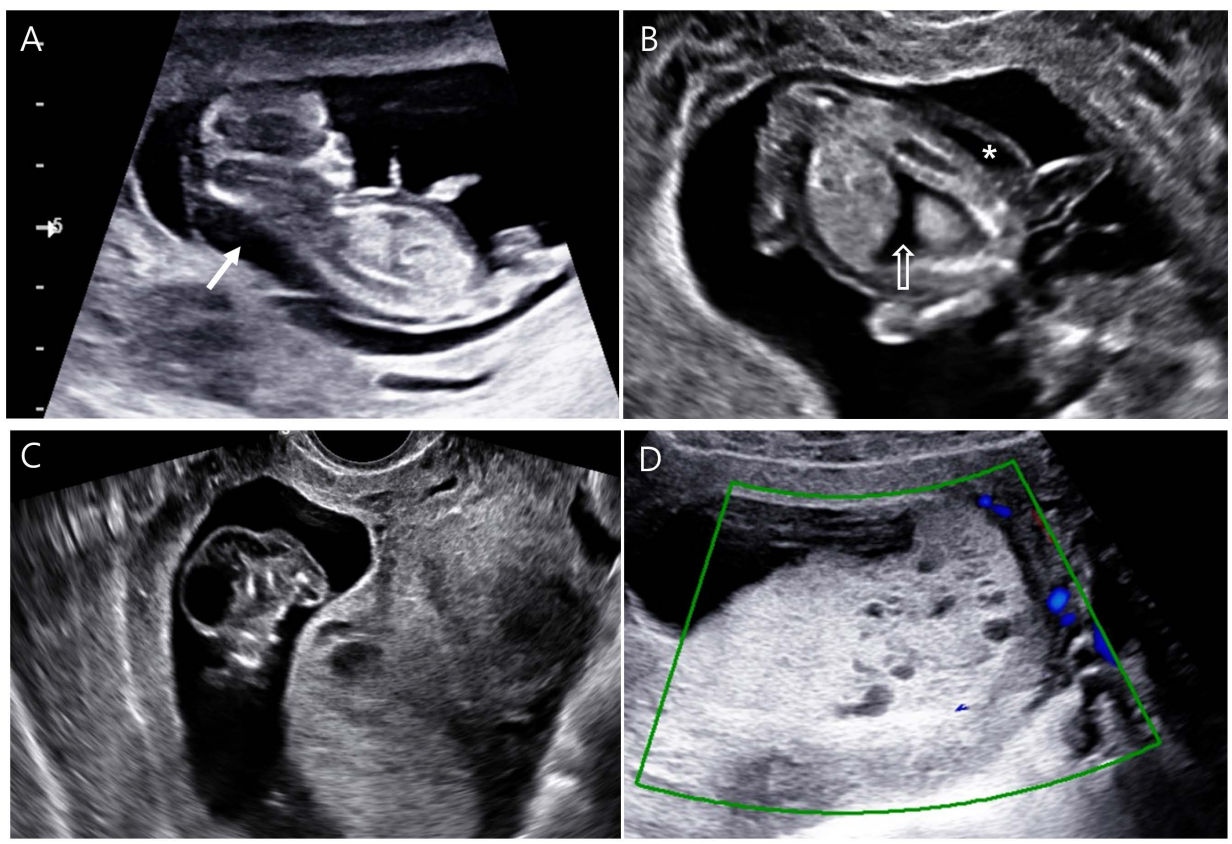

Fig. 1. Ultrasonographic findings at 10 weeks and 3 days of gestation. (A) Increased nuchal translucency (arrow). (B) Pleural effusion (open arrow) and skin edema (asterisk). (C) Fetal demise. (D) Placenta contained multiple hypoechoic cysts without blood flow admixed with normal tissue. 


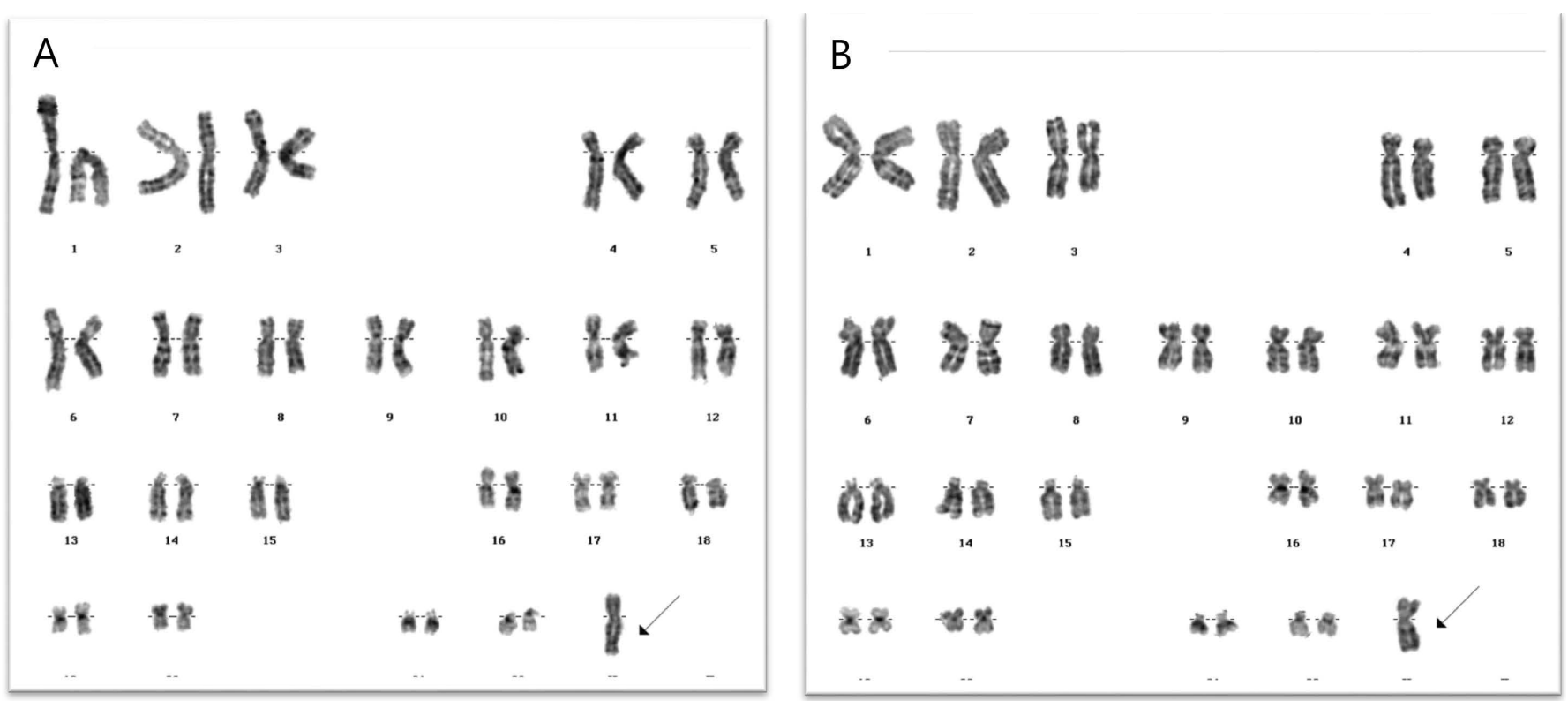

Fig. 2. (A) Karyotype of the fetus from chorionic villi showing 45,X (arrow). (B) Karyotype of the fetus from the amniotic fluid showing 45,X (arrow).

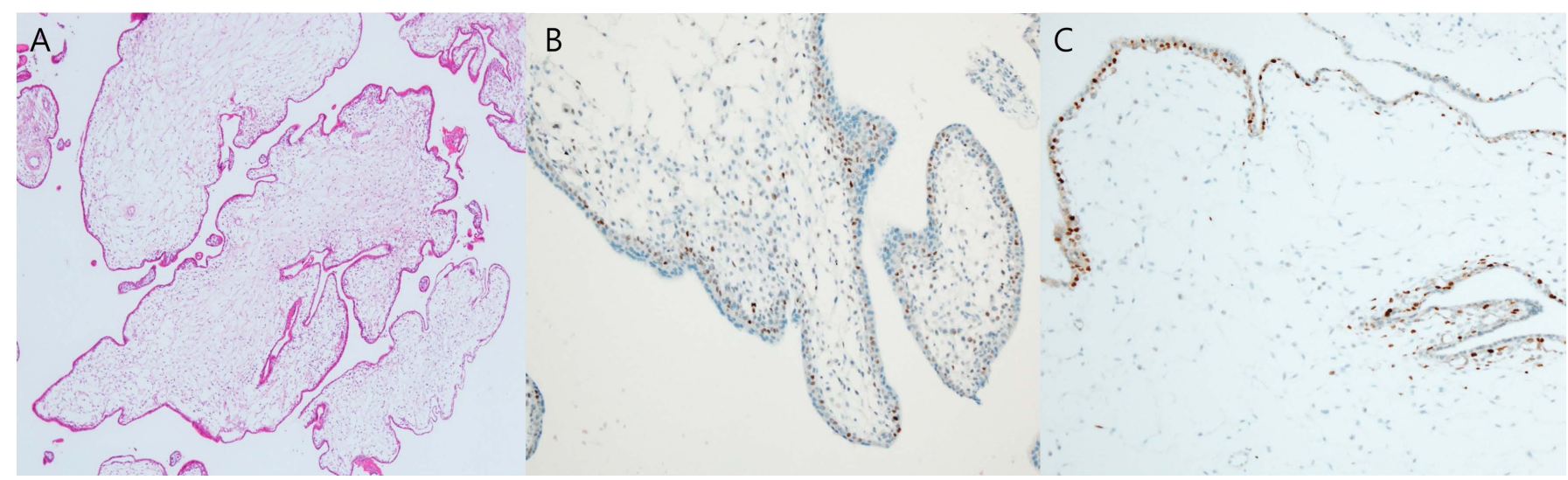

Fig. 3. Pathologic findings from the placenta. (A) Microscopic picture of the partial molar placenta showing a mixture of normal villi and molar villi displaying scalloped outlines (H\&E stain, $\times 40$ ). (B) Positive staining for p53 in the cytotrophoblast (Immunohistochemical stain, $\times 100)$. (C) Positive staining for $\mathrm{p} 57$ in the cytotrophoblast (Immunohistochemical stain, $\times 100)$.

paternally derived and the mole is considered androgenetic [6].

Most partial moles are triploid and accompanied by a fetus that has a triploid karyotype resulting from dispermic fertilization of a normal oocyte [7]. Therefore, the conceptus is mostly triploid with a biparental genome. In a partial molar pregnancy, the fetus can develop but is usually malformed and nonviable, while no fetus develops in a complete molar pregnancy. Although rare, there have been a few sporadic antenatally diagnosed cases of hydatidiform mole with a coexisting diploid live fetus $[3,8]$.

Hydatidiform mole with coexisting fetus carries an inherent risk of severe maternal complications, such as early-onset preeclampsia, thyrotoxicosis, antepartum hemorrhage, placenta accreta, gestational trophoblastic disease, and metastasis. In addition, intrauterine demise is common in such fetuses due to congenital anomalies and severe intrauterine fetal growth retardation, in turn caused by limited normal functional placental circulation $[9,10]$. These characteristics support the need for early diagnosis in the prenatal period.

Ultrasonography is a very useful diagnostic tool for prenatal diagnosis of hydatidiform mole with coexisting fetus. The diagnostic criteria on ultrasonography include a greatly enlarged placenta relative to the size of the uterine cavity, associated with cystic spaces. Other ultrasonographic findings linked with partial molar pregnancy include fetal malformation, growth restriction, and oligohydramnios. Recent advances in resolution have made 
diagnosis by ultrasonography in the first trimester of pregnancy possible, i.e., before the appearance of typical clinical symptoms. The diagnosis rate by ultrasonography differs according to gestational age at the time of diagnosis and subtype. In the first trimester of pregnancy, complete molar pregnancies have a diagnosis rate of 58-79\% while partial molar pregnancies have a diagnosis rate of $29-41 \%$. The overall diagnosis rate for molar pregnancies is $33-44 \%[11,12]$. Here, we found clusters of tiny cysts partially distributed in the placenta by ultrasonography at 10 weeks and 3 days of gestation, and suspected partial mole.

In cases of hydatidiform mole with coexisting fetus suspected based on the above ultrasonographic findings, amniocentesis or chorionic villous biopsy is performed to determine the karyotype, considering the high rate of poor perinatal outcome [13]. If the coexisting fetus has a normal karyotype, the maternal and fetal risks should first be explained to the mother and a decision made whether to continue pregnancy. We performed both chorionic villous biopsy and amniocentesis to avoid misdiagnosis due to confined placental mosaicism. Unfortunately, the limitations of our report are that we did not perform chorionic villous biopsy of the molar placenta due to procedure-related risk for bleeding. In addition, after delivery, conventional karyotyping was not feasible once the placental tissue was paraffin-embedded for histologic study. Therefore, chromosomal analysis of the partial mole was not performed and we were unable to firmly support our diagnosis.

The final diagnosis of hydatidiform mole is made based on histological findings. However, there is a limit to the morphological features of early molar pregnancy. Using immunochemical staining or DNA ploidy can facilitate accurate differential diagnosis between complete and partial mole.

Immunohistochemical staining for p57, a maternally expressed and paternally imprinted cyclin-dependent kinase inhibitor, is a potential marker that can help to distinguish between partial and complete moles or placental mesenchymal dysplasia [14]. In addition, expression of p53, one of the key regulators of apoptosis, can be used to distinguish partial hydatidiform mole from hydropic abortion [15]. In our case, we performed immunostaining for $p 53$ and p57 and positive results were obtained.

In this case, the coexisting fetus was found to have severe hydrops on ultrasound examination and had 45,X karyotype. However, if the coexisting fetus has a normal karyotype and ultrasonography reveals no fetal anomalies, optimal management is challenging and remains a dilemma; this is because these pregnancies are associated with maternal as well as fetal complications. The decision is more difficult if pregnancy has been achieved through in vitro fertilization. Immediate termination of pregnancy is typically recommended after diagnosis of hydatidiform mole with coexisting live fetus due to adverse maternal and fetal outcomes. However, several cases of live newborns with a diploid karyotype coexisting with a partial hydatidiform mole have been reported. Close maternal and fetal surveillance may help in achieving a favorable outcome, and termination may be required only when there is a gross fetal anomaly or deteriorating maternal condition [7].

The malignant potential of partial hydatidiform mole is still controversial. Persistent gestational trophoblastic disease arises in $14.5 \%$ of cases of partial hydatidiform mole, and despite evacuation, persistent gestational trophoblastic disease was reported in about 4-14\% of cases of partial hydatidiform mole $[16,17]$. It has also been reported that the distribution of diploid form of partial hydatidiform mole is more closely related to the development of persistent gestational trophoblastic disease than in triploid form $[18,19]$. In addition, sensitivity to chemotherapy is low in cases of the diploid form [20]. Therefore, further studies are required to evaluate the effects of diploid mating on the development of persistent gestational trophoblastic tumors. Combined placental histopathology, including immunohistochemical staining and placental karyotyping, may allow for more accurate diagnosis and prediction of prognosis. However, in this case, due to the absence of karyotype confirmation of the placenta post-delivery, we could not determine whether it was the diploid or triploid form of partial hydatidiform mole.

In conclusion, we reported a rare case of twin pregnancy with partial hydatidiform mole and coexistent fetus, which was diagnosed at 11 and 5/7 weeks of gestation. Insufficient information has been collected to allow valid prediction of outcome for patients with partial mole and coexistent fetus, due to its rarity, but careful prenatal sonography and karyotyping can facilitate early detection and may be helpful for achieving a favorable prognosis.

\section{References}

1. Jones WB, Lauersen NH. Hydatidiform mole with coexistent fetus. Am J Obstet Gynecol 1975;122:267-72.

2. Chu W, Chapman J, Persons DL, Fan F. Twin pregnancy with partial hydatidiform mole and coexistent fetus. Arch Pathol Lab Med 2004;128:1305-6.

3. Rathod S, Rani R, John LB, Samal SK. Successful outcome of twin gestation with partial mole and co-existing live fetus: a case report. J Clin Diagn Res 2015;9:0d01-2. 
4. Sebire NJ, Fisher RA, Rees HC. Histopathological diagnosis of partial and complete hydatidiform mole in the first trimester of pregnancy. Pediatr Dev Pathol 2003;6:69-77.

5. Szulman AE, Surti U. The syndromes of hydatidiform mole. II. Morphologic evolution of the complete and partial mole. Am J Obstet Gynecol 1978;132:20-7.

6. Kajii T, Ohama K. Androgenetic origin of hydatidiform mole. Nature 1977;268:633-4.

7. Guven ES, Ozturk N, Deveci S, Hizli D, Kandemir O, Dilbaz S. Partial molar pregnancy and coexisting fetus with diploid karyotype. J Matern Fetal Neonatal Med 2007;20:175-81.

8. Lee SW, Kim MY, Chung JH, Yang JH, Lee YH, Chun YK. Clinical findings of multiple pregnancy with a complete hydatidiform mole and coexisting fetus. J Ultrasound Med 2010;29:271-80.

9. Unsal MA, Guven S. Complete hydatidiform mole coexisting with a live fetus. Clin Exp Obstet Gynecol 2012;39:262-4.

10. Dolapcioglu K, Gungoren A, Hakverdi S, Hakverdi AU, Egilmez E. Twin pregnancy with a complete hydatidiform mole and co-existent live fetus: two case reports and review of the literature. Arch Gynecol Obstet 2009;279:431-6.

11. Fowler DJ, Lindsay I, Seckl MJ, Sebire NJ. Routine pre-evacuation ultrasound diagnosis of hydatidiform mole: experience of more than 1000 cases from a regional referral center. Ultrasound Obstet Gynecol 2006;27:56-60

12. Sebire NJ, Rees H, Paradinas F, Seckl M, Newlands E. The diagnostic implications of routine ultrasound examination in histologically confirmed early molar pregnancies. Ultrasound Obstet Gynecol 2001;18:662-5.

13. Sarno AP Jr, Moorman AJ, Kalousek DK. Partial molar pregnancy with fetal survival: an unusual example of confined placental mosaicism. Obstet Gynecol 1993;82(4 Pt 2 Suppl):716-9.

14. Hoffner L, Dunn J, Esposito N, Macpherson T, Surti U. P57KIP2 immunostaining and molecular cytogenetics: combined approach aids in diagnosis of morphologically challenging cases with molar phenotype and in detecting androgenetic cell lines in mosaic/chimeric conceptions. Hum Pathol 2008;39:63-72.

15. Kheradmand P, Goudarzi M, Tavakoli M. Analysis of p53 expression in partial hydatidiform mole and hydropic abortion. Front Bio 2017:12:357-60

16. Watson EJ, Hernandez E, Miyazawa K. Partial hydatidiform moles: a review. Obstet Gynecol Surv 1987;42:540-4.

17. Teng NN, Ballon SC. Partial hydatidiform mole with diploid karyotype: report of three cases. Am J Obstet Gynecol 1984;150:961-4.

18. Jauniaux E. Partial moles: from postnatal to prenatal diagnosis. Placenta 1999;20:379-88.

19. Davis JR, Kerrigan DP, Way DL, Weiner SA. Partial hydatidiform moles: deoxyribonucleic acid content and course. Am J Obstet Gynecol 1987;157(4 Pt 1):969-73.

20. Lage JM, Berkowitz RS, Rice LW, Goldstein DP, Bernstein MR, Weinberg DS. Flow cytometric analysis of DNA content in partial hydatidiform moles with persistent gestational trophoblastic tumor. Obstet Gynecol 1991;77:111-5. 